\title{
Searching for Biomolecules with the ATCA: First Results
}

\author{
Maria R Hunt-Cunningham \\ School of Physics, University of New South Wales, Sydney, NSW, 2052, \\ Australia. \\ Paul A Jones \\ Australia Telescope National Facility, CSIRO, PO Box 76, Epping, \\ NSW, Australia.
}

\begin{abstract}
A search for biologically significant molecules in the interstellar medium (ISM) is currently being undertaken with the Australia Telescope Compact Array (ATCA) of the CSIRO Australia Telescope National Facility (ATNF) at millimetre wavelengths. The search includes several chiral molecules, and glycine, the simplest amino acid. Some first results from this search are presented here.
\end{abstract}

\section{Introduction}

It is presumed that life on Earth evolved from an initial reservoir of simpler prebiotic organic material such as amino acids, fatty acids and sugars. What is not clear is whether this initial reservoir originated on the early Earth or in the presolar nebula. The detection of chiral molecules, in particular, in the interstellar medium (ISM) would be a persuasive argument in favour of an extraterrestrial origin for the initial reservoir of prebiotic molecules. Chiral molecules are those where asymmetry in carbon atom placement within a molecule leads to two distinct forms (mirror images or enantiomers).

Investigations of primitive mechanisms of self-replication that involve RNA only indicate the need for a reservoir of homochiral (having like chirality) organic molecules on the early Earth before living organisms could evolve (Joyce et al. 1984). An excess of about $10 \%$ in one enantiomer over the other ( $L$ over $D$ in the case of amino acids on the Earth) is needed for RNA template reproduction to work.

Non-biological processes always create chiral organic molecules in a racemic mix (equal numbers of both handednesses) and there are no known abiotic mechanisms operating on the surface of the Earth to generate the required enantiomeric excess. Therefore, an extraterrestrial origin for the prebiotic molecules needed to seed life on Earth is likely if we accept the hypothesis that primitive reproduction involved only RNA. Presumably the chiral molecules were delivered to the early Earth by a mechanism such as cometary or meteoritic bombardment.

An extraterrestrial mechanism for producing the requisite homochirality is necessary. The most likely mechanism is circularly polarized UV radiation in 
the ISM, which is known to be capable of generating an enantiomeric excess by selectively destroying one enantiomer over the other (Bailey et al 1998).

Despite the inferred extraterrestrial origin of homochiral molecules, none of the molecules so far detected in the ISM are chiral. The search for biologically important molecules with the ATCA is focusing on a number of chiral molecules for which simpler, non-chiral, but chemically related species have already been detected in the ISM. In addition, this search includes glycine. Glycine is the simplest amino acid utilized in biological processes on Earth although it is not a chiral molecule.

\section{Previous Searches for Glycine}

There have been many searches for glycine in molecular clouds, dating back over 20 years (e.g., Brown et al. 1979; Hollis et al. 1980; Snyder et al. 1983) with negative results. One of the most important recent searches was by Combes, Rieu, \& Wlodarczak (1996) who used the IRAM 30-m telescope (single dish) for the positions Orion $\mathrm{SiO}$ and $\mathrm{Sgr} \mathrm{B} 2(\mathrm{OH})$ and concluded that the confusion in the spectra of millimetre spectral lines, particularly in the Orion position is a major problem.

On the other hand, Miao et al. (1994) and Snyder (1997) report a possible detection of a $90 \mathrm{GHz}$ transition of glycine in Sgr B2 (N) with BIMA and OVRO (interferometers) There are some caveats in Snyder (1997) due to uncertainty in the glycine line rest frequency, the different velocity components in Sgr B2 $(\mathrm{N})$, and the confusion with other lines. If confirmed, the detection would give a column density $\mathrm{N}$ (glycine) $=5.9 \times 10^{15} \mathrm{~cm}^{-2}$, or if this is not confirmed, an upper limit $\mathrm{N}$ (glycine) of $<3 \times 10^{15} \mathrm{~cm}^{-2}$. The position Sgr B2 (N) contains the Large Molecule Heimat (LMH), a small diameter region that is particularly rich in large molecules.

Ceccarelli et al. (2000) have searched for glycine around the solar type protostar IRAS 16293-2422 with the IRAM 30-m telescope, arguing that low mass protostars may be more favourable than the massive protostars for glycine to be formed and survive. They derive an upper limit $\mathrm{N}$ (glycine) $<(0.7-1.5) \times 10^{13} \mathrm{~cm}^{-2}$.

\section{The ATCA Search for Biomolecules}

The ATCA is an excellent instrument for molecular line detection experiments, with five antennas of $22-\mathrm{m}$ diameter, giving a sensitivity somewhat better than comparable arrays at $3-\mathrm{mm}$ wavelengths, such as BIMA, OVRO, IRAM or Nobeyama which have smaller antennas.

The tentative detection of glycine by Snyder (1997) are for conformer I. This conformer has lower energy states than conformer II (from around $100 \mathrm{~cm}^{-1}$ above the ground state), however it also has a dipole moment that is a factor of 5.9 times lower than that of conformer II (Lovas et al. 1995). As intensity of emission from a transition varies as the dipole moment squared, the emission from transitions of conformer II may be up to 35 times stronger than those of conformer I, especially in a highly excited region such as the LMH in Sgr B2. 
This search is for the stronger conformer II transitions, utilising high frequency resolution, to minimise the problem of confusion from other lines. The search has a multi-pronged approach, involving both single dish and interferometer observations of multiple frequencies within the $3-\mathrm{mm}$ band.

Several molecular clouds will be targeted to maximise the chances of detection as it is not yet clear which are the best conditions for finding glycine. The target positions are Orion-KL, Sgr B2 (LMH) and IRAS 16562-3959.

The position Sgr B2 (LMH) is of small diameter ( $<5$ arcseconds) and has so far provided detections of the related complex molecules methyl formate and acetic acid (which is a precursor to glycine in some reaction networks). These molecules seem to be confined to the LMH. However, another similar molecule, glycolaldehyde, was found to be extended in the Sgr B2 (N) molecular cloud, with very low concentrations in the LMH. These three molecules are believed to have a common origin with glycine (Sorrell 2001) based on a model of chemical reactions in icy grain mantles. It is therefore important to search for glycine in both extended and compact warm, dense molecular gas.

In addition we are searching for glycine at high resolution towards the molecular cloud G345.5+1.5, which contains the IRAS point source 16562-3959, a bright, highly obscured infrared object. The relative molecular abundances for this object are particularly high, approaching those in Orion KL for some molecules, and it appears to contain a hot core (Hunt 2001). The lack of radio continuum emission suggests that any embedded star is of lower mass than $\mathrm{O}$ or $\mathrm{B}$, or is still in the protostar phase. G345.5+1.5 provides a contrasting environment in which to search for glycine. The lower UV flux compared to Sgr B2 may provide a more stable environment for glycine.

We are using both the ATCA and the single dish Mopra radio telescope, at Coonabarabran in northern NSW, Australia. The Mopra telescope, jointly operated by the Australia Telescope National Facility CSIRO, and the University of New South Wales, Australia, has a 22-m diameter, making it the largest single dish telescope operating at $3-\mathrm{mm}$ in the southern hemisphere.

\section{Preliminary Results}

Three 87-GHz transitions of conformer II glycine were observed with the ATCA in the molecular clouds Sgr B2 and G345.5+1.5 in 2002 June. A bandwidth of $64 \mathrm{MHz}$ divided into 128 channels was used. These data were obtained with the three ATCA 22-metre antennas fitted with the new 3 -mm system by 2002 . The complete $3-\mathrm{mm}$ system on five antennas will be installed by 2004 , giving even better performance.

Some results of these observations are shown in Fig. 1. The observing band was centred on a transition of glycine. Glycine is not detected in this spectrum; however, several other lines are seen (see figure caption). The rms noise in the spectra obtained from the data cube is around $30 \mathrm{mJy} /$ beam.

Further ATCA observations in 2002 August have covered another four 64$\mathrm{MHz}$ bands in Sgr B2(LMH). These have been complemented by observations with the Mopra telescope in 2002 August.

The June and August observations have together led to the detection of 16 previously unreported molecular lines in Sgr B2. 


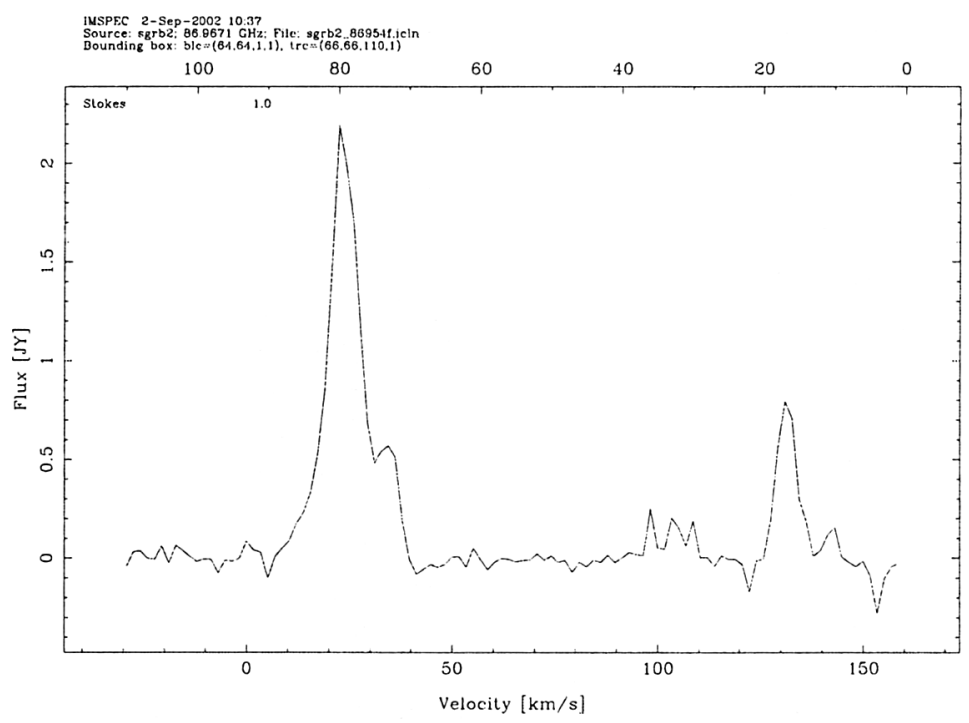

Figure 1. The 87-GHz spectrum towards Sgr B2 (LMH). The line near channel 80 (top scale)is unknown line U86980 (Turner 1989). The three lines near channel 35 and the line near channel 15 have not previously been reported.

\section{References}

Bailey, J., Chrysostomou, A., Hough, J. H., Gledhill, T. M., McCall, A., Clark, S., Menard, F. \& Tamura, M. 1998, Science, 281, 672

Brown, R. D., et al. 1979, MNRAS, 186, 5

Ceccarelli, C., Loinard, L., Castets, A., Faure, A. \& Lefloch, B. 2000, A\&A, 362, 1122

Combes, F., Rieu, N. Q., \& Wlodarczak, G. 1996, A\&A, 318, 618

Hollis, J. M., Snyder, L. E., Suenram, \& R. D., Lovas, F. J. 1980, ApJ, 241, 1001

Hunt, M.R. 2001, PhD thesis, University of Western Sydney

Joyce, G. et al. 1984, Nature, 310, 602

Lovas, F. J., Kawashima, Y., Grabow, J-U, Suenran, R. D., Fraser, G. T. \& Hirota, E. 1995, ApJ, 455, L201

Miao, Y., Snyder, L. E., Kuan Y.-J., \& Lovas, F. J. 1994, Bull. Am. Astron. Soc., 26, 906

Snyder, L. E. 1997, Origins of Life and Evolution of the Biosphere, 27, 115

Snyder, L. E., Hollis, J. M., Suenram, R. D., Lovas, F. J., Brown, L. W., \& Buhl, D. 1983, ApJ, 268, 123

Sorrell, W. H. 2001, ApJ, 555, L129

Turner, B. E. 1989, ApJS, 70, 539 